\title{
A serial sample processing strategy with improved performance for in-depth quantitative analysis of type III secretion events in Pseudomonas aeruginosa
}

\begin{abstract}
Dimitrios Lampaki ${ }^{1,2}$, Andreas Diepold ${ }^{2 *}$, Timo Glatter ${ }^{1 *}$
${ }^{1}$ Core Facility for Mass Spectrometry and Proteomics, Max Planck Institute for Terrestrial Microbiology, Karl-von-Frisch-Str. 10, D-35043, Marburg, Germany

${ }^{2}$ Department of Ecophysiology, Max Planck Institute for Terrestrial Microbiology, Karl-vonFrisch-Str. 10, D-35043, Marburg, Germany
\end{abstract}

*Corresponding authors

Dr. Timo Glatter

Tel: +49 (6421) 178 334; Fax: +49 (6421) 178 209;

E-mail: timo.glatter@mpi-marburg.mpg.de

Dr. Andreas Diepold

Tel: +49 (6421) 178 302; Fax: +49 (6421) 178 209;

E-mail: andreas.diepold@mpi-marburg.mpg.de 


\section{TABLE OF CONTENTS}

1. Supplementary figures (within one pdf file).

Figure S-1. Charge state intensity distribution of the different secretome worflows.......S-3

2. Supplementary tables (in separate excel sheet (xlsx))

Supplementary Table S-1: Spectrum counts (Scaffold export) with associated number MS/MS queries identifying Pseudomonas aeruginosa proteins from different supernatant preparation workflows.

Supplementary Table S-2: LFQ peptide ion MS1 and protein quantification of WT (PAO1) versus delta $p s c V$ processed with the TCA workflow.

Supplementary Table S-3: LFQ peptide ion MS1 and protein quantification of WT (PAO1) versus delta $p s c V$ processed with the SP3 workflow.

Supplementary Table S-4: LFQ peptide ion MS1 and protein quantification of WT (PAO1) versus delta $p s c V$ processed with the TCA-SP3 workflow.

Supplementary Table S-5: Spectrum counts of identified proteins over the secretion time course (Scaffold export).

Supplementary Table S-6: iBAQ values derived from MaxQuant over the secretion time course. 
Precursor charge state intensity distribution
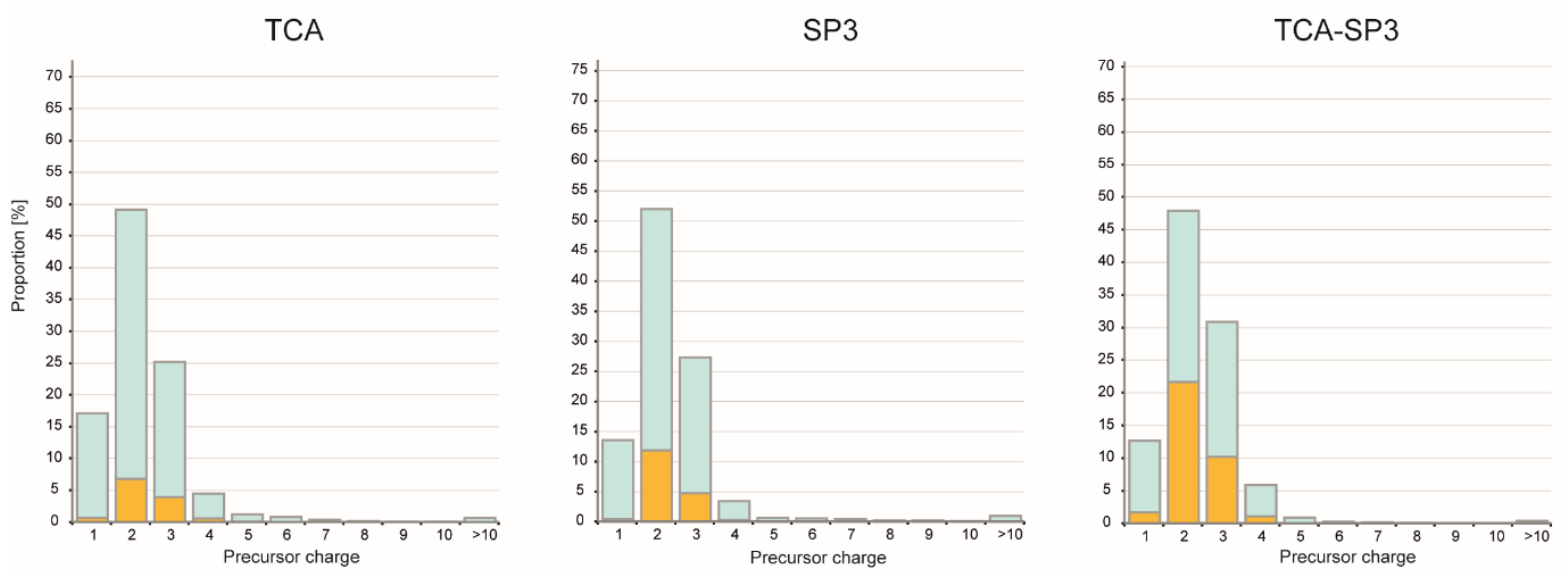

Figure S-1. Charge state intensity distribution of the different secretome worflows. Upon sample processing, LC-MS measurement and label-free quantification, the MS1 ion charge-state distribution was exported from Progenesis. The fraction of identified versus not identified ions is color-coded. 